\title{
Therapeutic Role of Coenzyme Q10 or/and $\alpha$-lipoic acid on Cardiac Functions in Obese/Hypertensive Rats
}

\author{
Shawky, M.F.;* Wahba, S.R. *and Heibashy, M.I.** \\ *Zoology Department, Faculty of Women for Arts, Science and Education, Ain Shams \\ University. \\ **Biological Applications Department, Radioisotopes Applications Division, Nuclear Research \\ Center, Atomic Energy Authority, Egypt.
}

\begin{abstract}
This study focuses on the relationship between obesity and hypertension and the possible amelioration effects of co-enzyme Q10 (CoQ10) or/and $\alpha$-lipoic acid (ALA) and their mixture on histological and physiological changes in rat heart.

To achieve this purpose, a comparison took place between normal control rats group (20 rats) and obese/hypertensive rats group (24 rats). The obese/hypertensive rats were injected (i.v) with Poloxamer-407(P-407) as a single dose (1g/kg b.wt dissolved in $1 \mathrm{ml}$ cold saline) to induce obesity and with the aid of oro-gastric tube $1 \mathrm{~g}$ sodium chloride salt/kg b.wt/day for 30 days for induction of hypertension. Control rats group (20 rats) were divided into 5 animals (normal control rats subgroup), 5 animals treated with 200mg CoQ10/kg b.wt /day (Normal + CoQ10 rats), 5 rats treated with $100 \mathrm{mg}$ ALA/kg b.wt /day (Normal + ALA rats) and the last 5 animals treated with both antioxidants as the above subgroups (Normal + CoQ10 + ALA) by oro-gastric tube. The 24 obese/hypertensive rats were divided to four subgroups, the first subgroup (6 obese/hypertensive rats), the second subgroup (6 rats) was treated with $200 \mathrm{mg}$ CoQ10/kg/b.wt/day (obese/hypertensive rats + CoQ10 rats), the third (6 rats) was treated with $100 \mathrm{mg} \mathrm{ALA} / \mathrm{kg}$ b.wt/day (obese/hypertensive rats + ALA rats) and the last 6 animals were treated with both antioxidants as above described (obese/hypertensive rats + CoQ10 + ALA). All rats were dissected after 4 week experimental duration.

Histological alterations in cardiac tissue of obese/hypertensive rats included lack of striation of myocytes with pyknotic nuclei; interstitial edema; congested blood vessels and mononuclear cellular infiltration in expanded intracellular spaces. After the induction of obesity and hypertension in rats and in comparison to normal control animals, the results showed significant $(\mathrm{P}<0.05)$ increase in serum concentrations of cholesterol, triglycerides, HDLcholesterol and LDL-cholesterol. Also, remarkable increments in the serum heart enzymes activities of creatine kinase (CK), creatine kinase -MB (CK-MB), lactate dehydrogenase (LDH) and aspartate aminotransferase (AST).
\end{abstract}

\section{*Correspondent author: monafathy.mona1993@gmail.com.}


The increment of serum levels of heart fatty acid binding protein (H-FABP) and endotheline-1 (ET-1) occurred. On the other hand, a significant $(\mathrm{P}<0.05)$ decrease in the level of serum total nitric oxide was recorded in obese/hypertensive rats compared with those corresponding normal control ones.

When obese/hypertensive rats subgroups were treated with CoQ10 or/and ALA, considerable ameliorative effects in all previous studied parameters were pronounced dependent on certain mechanisms discussed according to available recent researches. Moreover, histological studies of the heart also revealed a definite ameliorative effect of these antioxidants as regards tissue damage and structural integrity.

Keywords: Obesity/hypertension, Poloxamer 407, Sodium chloride salt, CoQ10, ALA, Rats.

\section{INTRODUCTION}

Obesity is a multi-factorial process with complex interactions among genetic, metabolic, hormonal, and psychological factors. Physiologically, obesity is an imbalance resulting from the failure of the coupling between intake and expenditure (Krishnan et al., 2007 and WHO, 2017). Obesity is characterized by hyperlepidemia, and resistance of hypothalamic satiety center to anorectic effect of adipose tissue hormone (Heibashy et al., 2010 and Karri et al., 2019).

Also, hypertension is a common problem facing man today. Because high blood pressure is one of the leading causes of stroke and a major risk factor for heart attack, one of the most important aspects of preventive cardiology should be to identify as many people who have the disease as possible and to take steps to lower the blood pressure before it causes damage to the blood vessels, heart, kidneys, eyes and other organs (Samavat \& Hojjatzade, 2012 and Mahmoud et al., 2016). Fortunately, the last decades have seen enormous amount of scientific researches that dealt with supplementation of specific nutrients which showed remarkable advances in the treatment of high blood pressure. Hence, this study was focused on dietary approaches of hypertension induced artificially in animal models (Heibashy \& abdel Moniem, 2005; Kitada et al., 2017 and Lanaspa et al., 2018).

Co-enzyme Q10 (CO Q10) is a powerful antioxidant. It is a naturally occurring vitamin present in nearly all tissues (Sander et al., 2006). Several studies have clearly shown the potential benefit of co-enzyme Q10 in treatment of hypertension and congestive heart failure (Heibashy et al., 2014 and Song et al., 2017). Other authors found that coenzyme Q10 may also be effective in reducing total cholesterol (Tsuneki et al., 2007; McMurray et al., 2010 and Sharma et al., 2016). They indicated that CoQ10 can efficiently prevent high glucose induced endothelial cell apoptosis and adhesion to monocytes, which are relevant to the pathogenesis of atherosclerosis.

Moreover, it was found that CoQ10 levels of patients who have chronic cardiac failure are too low both in tissue and serum samples (Venkat et al. 2009). CoQ10 provides improvement in treatment of congestive cardiac failure, stroke and cardiac fraction (Sander et al., 2006 and Song et al., 2017). 
CoQ10 also reduced elevated blood pressure, but did not affect body weight gain in cases of metabolic syndrome. In addition, CoQ10 improved endothelial dysfunction in the mesenteric arteries suggesting that the antioxidant properties of CoQ10 can be effective in ameliorating cardiovascular risk in metabolic syndrome (Tiano et al., 2007 and Bekir et al., 2016).

$\alpha$-lipoic acid (ALA) acts by multiple mechanisms both physiologically and pharmacologically. Its pharmacological role is to improve glycemic control and prevent polyneuropathy. Physiologically, it acts as an antioxidant by directly terminating free radicals (Zhang et al., 2007 and Guofu et al., 2014). So, several studies have documented its positive therapeutic effect, particularly in diseases such as diabetes, atherosclerosis and neurodegenerative diseases (Papanas \& Ziegler, 2014 and Ambrosi et al., 2018).

The current investigation was undertaken to validate the possible effectiveness of the abovementioned nutrients (Coenzyme Q10 and $\alpha$-lipoic acid) to adjust the disturbances in certain blood biochemical components and changes in histological heart studies reflecting some aspects of obesity and heart troubles associated with high blood pressure.

\section{MATERIAL AND METHODS}

Forty-four adult male albino rats (Rattus rattus) approximately of the same age $(10 \pm 1$ week old) and weight $(130 \pm 10 \mathrm{~g})$ were obtained from the Breeding unit, Serum and Antigen Laboratories at Helwan and employed in this investigation. They were caged and provided with diet and tap water ad libitum for one week prior to the experiment for adaptation.

Experimental protocols followed the Guidelines for the Care and Use of Laboratory Animals approved by the Institutional Ethics Committee of Ain Shams University. The obese/hypertensive rats were injected (i.v) with Poloxamer-407 (P-407) as a single dose (1g/kg b.wt dissolved in $1 \mathrm{ml}$ cold saline) according to Chaudhary \& Brocks (2013) to induce obesity and with the aid of oro-gastric tube $1 \mathrm{~g}$ sodium chloride salt/kg b.wt/day for 30 days according to Kitada et al. (2017) for induction of hypertension. The control rats group (20 rats) were divided into 5 animals (normal control rats subgroup), 5 animals treated by oro-gastric tube with $200 \mathrm{mg}$ CoQ10/kg b.wt /day (Khatta et al., 2000) (Normal + CoQ10 rats), 5 rats treated by oro-gastric tube with 100mg ALA/kg b.wt /day (Goraca et al., 2011) (Normal + ALA rats) and the last 5 animals treated with both antioxidants as above subgroups (Normal + CoQ10 + ALA). The 24 obese/hypertensive rats were divided to four subgroups, the first subgroup (6 rats obese/hypertensive rats), the second subgroup of obese/hypertensive rats (6 rats) was treated with 200mg CoQ10/kg/b.wt/day (obese/hypertensive rats + CoQ10 rats), the third subgroup of obese/hypertensive rats (6 rats) treated with 100mg ALA/kg b.wt/day (obese/hypertensive rats + ALA rats) and the last 6 animals treated with both antioxidants as above described (obese/hypertensive rats + CoQ10 + ALA). All rats were dissected after 4 week experimental duration.

At the end of experimental period, rats were slightly anaesthetized by diethyl ether and sacrificed. Blood samples were immediately collected from the heart in clean dry test tubes and centrifuged at $10,000 \mathrm{rpm}$ for 20 minutes. Sera were separated and kept at $-20^{\circ} \mathrm{C}$ for the 
biochemical parameters. Hearts were carefully removed aseptically, washed with cooling saline solution $(0.9 \% \mathrm{NaCl})$ and placed in neutral buffer formalin for histopathological studies using the routine staining (haematoxylin and eosin).

Biochemical parameters included lipids profile: Serum total cholesterol (T-CH) Allain et al. (1974), triglycerides (TG) Fossati et al. (1982) and HDL-cholesterol (HDL-C) estimated enzymatically Grove (1979). LDL-cholesterol was calculated as peaccording to Assman's equation (Assman et al., 1984).

LDL = Total serum cholesterol - [serum triglyceride/5 -serum HDL- cholesterol].

Heart enzymes activities: Serum creatine kinase (CK) Fisher et al. (1983), creatine kinase -MB (CK-мB) Griffiths et al., (1977), lactate dehydrogenase (LDH) Buhl \& Jackson (1978) and aspartate aminotransferase (AST) IFCC (1978) activities were esssatimated kinetically.

Cardiac profile: serum heart fatty acid binding protein (H-FABP) Pagani et al. (2002) and endotheline-1 (ET-1) Wakisaka et al. (1996) levels were assayed using commercial ELISA (Sandwich Immunoassay Technique) specific kit for rats (Kamiya Biomedical Company, USA). Serum total nitric oxide (TNO) Griess et al. (1982) level was assayed using commercial ELISA technique kit form Cayman Chemical Company (USA).

Data were statistically analyzed using Two Way Analysis of Variance (ANOVA) by the aid of SPSS (version 20.0) program. Values were considered statistically significant when $\mathrm{p} \leq 0.05$.

\section{RESULTS}

\section{Histological studies:}

Cardiac sections taken from control rats manifested normal histological architecture of the myocardium (Fig. 1 a) with longitudinally striated branching and anastomosing muscle fibers with centrally located oval vesicular nuclei (Fig 1 b). Sections obtained from hyperlipidemic/hypertensive rats designated eosinophilic cardiac muscle fibres with lack of striation and pyknotic nuclei (Fig. 1 c). Also present in the interstitial tissue was nuclear fragments where necrotic muscle fibres were replaced by fibrous tissue. Mostly evident was lysis of groups of muscle fibres; interstitial edema; increased intracellular spaces between myocardial fibers invaded by nuclear fragments (Fig. $1 \mathrm{~d}$ ). Congested blood vessels were prominent with attenuated elastic fibres replaced by reticular architecture and reduced tunica media layers (Fig. 1 e). Mononuclear cellular infiltration in-between muscle fiber was also a prominent feature .

Following treatment of hyperlipidemic/hypertensive rats with either CoQ10 or ALA cardiac sections manifested regenerative profiles (Fig 2 a) with lesser appearance of degenerative necrotic cardiac cells (Fig. 2 b). With the double treatment with CoQ10 and ALA necrotic remnants in interstitial tissue was minimal with oppressed interstitial edema (Fig. 2 c \& d). Blood vessels partially regained normal appearance (Fig 2 e). 


\section{Biochemical parameters: \\ 1- Lipids profile:}

According to the obtained data in Table $(1)$, there was a significant $(\mathrm{p}<0.05)$ elevation in the levels of serum lipid profile (cholesterol, triglyceride, HDL and LDL) in obese/hypertensive rats group compared to their corresponding normal control. A remarkable correction occurred in the previous parameters after obese/hypertensive rats were treated with Co Q10 or ALA after 4 weeks treatment. The best corrections were reported in serum levels of T-Ch, TG, HDL-C and LDLC of obese/hypertensive rats group treated with both antioxidants (Co Q10 and ALA) compared to their corresponding normal control rats group (Table 1).

\section{2- Heart enzymes activities:}

Induction of obesity/hypertension in rats led to a significant $(\mathrm{p}<0.05)$ elevation in serum heart enzymes activities [creatine kinase (CK), creatine kinase -MB (CK-MB), lactate dehydrogenase (LDH) and aspartate aminotransferase (AST) as shown in Table (2). Treated obese/hypertensive rats with $200 \mathrm{mg}$ CoQ10/kg/b.wt/day or $100 \mathrm{mg}$ ALA/kg b.wt/day led to considerable amelioration effects on prementioned enzymes activities. Best amelioration effects were reported after obese/hypertensive rats were treated with both antioxidants (Co Q10 and ALA) compared to their corresponding normal control 4 weeks interval (Table 1).

\section{3- Cardiac profile:}

The obtained data in Table (3) clarified a significant $(\mathrm{P}<0.05)$ elevation in serum hearttype fatty acid binding protein (H-FABP) and endothelial-1 (Et-1) levels in obese/hypertensive rats However, the induction of obesity/hypertension in the animals caused a significant $(\mathrm{P}<0.05)$ decrease in serum total nitric oxide (TNO) level (Table 3).

Treated obese/hypertensive rats with $200 \mathrm{mg}$ CoQ10/kg/b.wt/day or $100 \mathrm{mg}$ ALA/kg b.wt/day led to considerable modulation effects on H-FABP, Et-1 and TNO levels (Table 3). Again maximum corrections were recorded as obese/hypertensive rats were treated with a mixture of Co Q10 and ALA compared to their corresponding normal control rats group as shown in Table (3).

\section{DISCUSSION}

Obesity is the most common nutritional disorder that is associated with increased mortality and morbidity of cardiovascular disease (WHO, 2017). On the other hand hyperlipidemia caused by elevation of cholesterol levels is a major risk factor in the incidence and pathogenesis of degeneration diseases including atherosclerosis and cardiovascular disease (Karri et al., 2019). In rats, P-407 administration as a single or repeated dose causes significant elevation in serum total cholesterol and triglycerides thus inducing hyperlipidemia (Korolenko, 2016).

Hypertension remains a common and serious problem, contributing in a major way to the most common causes of morbidity and mortality worldwide (Hulanicka et al., 2007) where high salt intake is one of the major risk factors for developing hypertension (Youshionwa et al., 
2004). Simonds et al. (2014) have proposed that leptin mediates the increase in blood pressure associated with obesity.

Presently, administration of both $\mathrm{P}-407$ and $\mathrm{NaCl}$ to induce hyperlipidemia/hypertension in rats caused a marked increment in serum $\mathrm{T}$ Ch, TG, HDL-C and LDL-C concentrations as compared to their corresponding control. These results may be due to the increase in free radicals production which led to alteration in the mitochondrial function (decrease in number and size and shape deformation) associated with alteration in the function of smooth ER due to the disturbance in the lipid oxidation. It may also be due to the increment in the de novo lipogenesis, disturbance in the hypothalamus-pituitary-thyroid axis (HPTA), alteration in the neuropeptide hormones (neuropeptide-Y, orexin-A and orexin-B) levels or/and the decrease of $\beta$-oxidation of lipid in the matrix of mitochondria. These results are in agreement with the viewpoint that mitochondrial dysfunction in the pathogenesis of hyperlipidemia as well as hypertension at different degrees mainly include lipid oxidation impairment and the induction of peroxidative production (Heibashy, 2005; Tanaka et al., 2009; Heibashy et al., 2013; Korolenko, 2016 and Rana et al., 2017).

Enzymes of significance for diagnosis of cardiac conditions i.e. cardiac enzymes include CK, LDH and AST. CK values rise 4 to 6 hours after cardiac infarction. They reach peak in 24 to 36 hours. AST values start rising at this time and reach peak values in 2 to 3 days. They stay elevated for about 14 days (Choi et al., 2016).

It was obvious in the present investigation that induced myocardial dysfunction as a result of pronounced hyperlipidemia/hypertension in rats led to a significant $(p<0.05)$ elevation in the serum heart enzyme activities (CK, CK-MB, LDH and AST) as compared to their corresponding control rats group.

These results may be due to excessive myocardial infarction as a result of increased production of reactive oxygen intermediates, a resultant rise in lipid peroxidation, epigenetic gene alteration, inhibition in activity of total nitric synathase (NOs) enzyme, increase in the level of serum endothelin-1 accompanied with elevation in serum asymmetric dimethyl arginine (ADMA) level as well as appearance of hypertension and metabolic syndrome. These results are in parallel with those obtained by Tanaka et al. (2009) and Korolenko et al. (2013 \& 2016). The authors attributed these disturbances to the loss of cardiac troponin from myocytes and myofibrillar lyses in the plasma membrane.

Due to cellular damage and cardiac infarction LDH leaks into the serum. As height and duration of the increased activity depends on the size of lesion therefore, LDH is used as an index of membrane damage. Also, Lau, (2018) found that when vascular endothelial cells were exposed to oxidized LDL, there was a significant increase of LDH release indicating cell membrane injury and disturbance in the lipid peroxidation of cardiac tissues (Faulx et al., 2005). Heibashy \& Abdel-Moneim, (2005) explained the increment of cardiac enzymes to the elevation of cardiac troponin level which mediates the calcium activation of contraction of cardiac muscle. 
Heart fatty acid binding protein (H-FABP) is an early and sensitive marker for myocardial infarction and myocardial injury Heibashy et al., 2013; Willemsen et al., 2015 and Vupputuri et al. (2015). Plasma level of endothelin-1 (ET-1) is elevated in obese or hypertensive patients. Nitric oxide (NO) is an important bioactive substance which plays an important role in the regulation of normal body function and disease occurrence (Brealey $\boldsymbol{e t}$ al., 2004).

Induced hyperlipidemia/hypertension in rats impair the integrity of the endothelial barrier resulting in endothelial cell injury, which contributes to greater permeability of endothelial cells and impaired homeostasis, and induces the release of cytokines and reactive oxygen species (ROS). Plasma level of endothelin-1 (ET-1) is elevated in obese or hypertensive patients associated with the severity of the illness (Heibashy et al., 2013; Willemsen et al., 2015 and Vupputuri et al., 2015). Also, Klotz et al. (2006) noted that endothelin-1 (ET-1) is a potent endogenous vasoconstrictor, mainly produced by endothelial cells.

In the current work, it was shown that after cardiac injury from induced hyperlipidemia/hypertension in rats serum levels of H-FABP and ET-1 were increased significantly $(\mathrm{p}<0.001)$ as compared to their corresponding control. These data may be attributed to the inhibition of nucleic acids as well as protein synthesis; increment of free radical production and oxidative stress associated with release of vasoactive amines; changes in adrenergic functions; abnormalities in the mitochondria; lysosomal alterations; disturbance in sarcolemma and membrane bound enzymes activities; alteration in the myocardial electrolytes and occurrence of $\mathrm{Ca}^{2+}$ overload; Kitada et al., 2017 and Lanaspa et al., 2018).

Currently it was shown that induction of hyperlipidemia/hypertension in rats group led to a remarkable decrease in the serum level of total nitric oxide (TNO) compared to their corresponding control rats group. These data may be due to increment in the oxidative damage, inflammation and apoptosis appearance as well as alterations in endothelial scavenger receptor class-B member-1 (SR-B1), phosphoinosotide-3 kinase (PI3K), mitogen activated protein kinases (MAPK) and protein kinase-B activity (Akt) associated with mutation of HDL-cholesterol production. These results are in harmony with several recent investigations (Heibashy \& AbdelMoneim, 2005; Ramanathan \& Thekkumalai, 2014; Kitada et al., 2017 and Vanhoutte, 2018).

To study the effect of $\mathrm{P}-407$ and $\mathrm{NaCl}$ salt on the structure of rat myocardium, sections were examined. Cardiac sections obtained from hyperlipidemic/hypertensive rats designated several pathological findings including eosinophilic cardiac muscle fibres with lack of striation and pyknotic nuclei; lysis of groups of muscle fibres; interstitial edema; increased intracellular spaces between myocardial fibers invaded by nuclear fragments; congested blood vessels with attenuated elastic fibres replaced by reticular architecture and karyolitic cardiac muscles. In addition, areas of pale homogeneous acidophilic cytoplasm were observed with either degenerative or deeply stained pyknotic nuclei. Mononuclear cellular infiltration in-between 
muscle fiber was also a prominent feature. These data are in accordance with those obtained by Brahmbhatt and Cowie, (2018); Saidu et al. (2018); Khallaf and El-Mansy, (2019).

Obesity is usually associated with increased myocardial oxygen consumption, increased fatty acid metabolism and thus reduced myocardial efficiency. Increased oxygen consumption is also a normal sequel of hypertension. Present histological alterations may be primarily attributed to oxidative stress experienced by cardiac cells resulting in an imbalance in the production of ROS and intrinsic antioxidant defense. This may lead to inflammatory reactions and fibroblast activation. Cardiac fibroblasts on the other hand may change into myofibroblasts to secrete extracellular matrix components as collagen, fibronectin and laminin finally leading to fibrosis. In addition, degenerative necrotic changes alter the ejection of sufficient blood during systole leading to dilatation of the heart and stretching of the muscle fibres and fall of cardiac output.

Heart failure (HF) may cause severe damage to the heart muscle via myocardial fibrosis, ventricular remodeling, decreased contractility, and increased myocyte apoptosis (Madamanchi \& Runge, 2013). Similar results were achieved in vitro (Ziegler et al., 2011 and Papanas \& Ziegler, 2014).

The current investigation was designed to evaluate the therapeutic role of coenzyme Q10 (CoQ10) or $\alpha$-lipoic acid (ALA) as well as their mixture on hyperlipidemic/hypertensive rats and their modulation effects on the myocardial dysfunction.

Coenzyme Q10 (CoQ10) also known as ubiquinone is a fat-soluble molecule found primarily in the mitochondrial inner membrane, functions as a physiologically essential electron carrier in the electron transport chain bucket brigade. When electrons have been added to CoQ10, the molecule is known as "ubiquinol"; in this "reduced" form, CoQ10 can donate electrons to free radicals, quenching them. The oxidant scavenging activity of ubiquinol is versatile and important; in particular, ubiquinol can quench peroxynitrite-derived radicals, protecting the proteins of the electron transport chain from peroxynitrite-mediated damage. Ubiquinol also helps to prevent the peroxidation of fats in mitochondrial membranes. The high utility of CoQ10 as a mitochondrial antioxidant reflects the fact, that after it has donated an electron to quench a free radical, the electron transport chain "reloads" it with another electron, so that it is once again primed to scavenge oxidants (Rodick et al., 2018).

Other researches indicated that CoQ10 may aid the protective function of the endothelial lining of arteries throughout reflecting a role for mitochondrial oxidant production in endothelial dysfunction (Hodgson \& Watts, 2003 and Tiano et al., 2007). In rodent studies, preadministration of CoQ10 has a favorable impact on the damage to cardiac tissue evoked by a temporary cessation of blood flow and decreased the oxidative stress in ischemia-reperfusion damage (Littarru et al., 2007).

In the current study, the supplementation of (COQ10) to hyperlipidemic/hypertensive rats caused marked amelioration effects in all studied including histological profile. 
The amelioration effects on current results may be due to the antioxidant action of CoQ10 which improves cardiac bioenergetics; has direct free radical scavenger and antioxidant effect; facilitate the synthesis of depleted glutathione; corrects coenzyme Q10 deficiency state; improves endothelial function and vasodilatory effect; increases the blood flow by increasing the soluble nitric oxide activity; has membrane-stabilizing activity due to phospholipid protein interactions; preserver of myocardial $\mathrm{Na}^{+}-\mathrm{K}^{+}$ATPase activity; stabilizes the integrity of $\mathrm{Ca}^{2+}$ channels with correction mitochondrial leak of electrons during oxidative respiration and improves the immune response system. The current data are in harmony with those obtained by Tsai et al. (2012); Yang et al. (2015); Acosta et al. (2016) and Song et al. (2017).

$\alpha$-lipoic acid (ALA), on the other hand, known as thioctic acid, is a naturally occurring compound that is synthesized by plants and animals, including humans (Yi \& Maeda, 2005). It contains two sulfur molecules that can be oxidized or reduced. This feature allows ALA to function as a cofactor for several important enzymes as well as a potent antioxidant (Özkan $\boldsymbol{e t}$ al., 2005).

ALA exerts its effect mainly through its reduced form, dihydrolipoic acid (DHLA), a conversion to which is catalyzed by lipoamide dehydrogenase. This endogenous thiol antioxidant quenches reactive oxygen species (singlet oxygen, $\mathrm{H}_{2} \mathrm{O}_{2}$, hydroxyl radicals), regenerates reduced glutathione (GSH) and chelates metals such as iron, copper, mercury and cadmium, which are known to mediate free-radical production in biological systems. Both ALA and DHLA also protect the integrity of cell membranes by interacting with other antioxidants e.g; glutathione and vitamins $\mathrm{E}$ or $\mathrm{C}$.

ALA being a very effective antioxidant is used in various diseases concerning agedependent oxidative stress (Rochette $\boldsymbol{e t}$ al., 2013). Also, ALA acting as a cofactor for enzymatic reactions within the mitochondria can improve mitochondrial function by conserving cellular energy (Packer \& Cadenas, 2011). Some studies on animal models have confirmed that ALA can prevent progressive remodeling and even improve cardiac function (Papanas \& Ziegler, 2014 Ambrosi et al., 2018 and Genazzani et al., 2018). They suggested that the cardioprotective effects of ALA are obtained by immunoreactive insulin enzyme through a mechanism involving the activation of this enzyme.

In the concurrent investigation, the supplementation of ALA to hyperlipidemia/hypertensive rats caused considerable correction effects in all studied parameters. These data are confirmed but to lesser degree than hyperlipidemic/hypertensive rats treated with CoQ10. These effects may be attributed to reduction in oxidative stress induced by both P-407 and $\mathrm{NaCl}$ by alleviating lipid peroxidation through free radical scavenging or by enhancing the synthesis of antioxidants containing -SH groups and GSH which then detoxify free radicals. Current results are in agreement with several recent authors (Rochette et al., 2013; Papanas \& Ziegler, 2014; Skibska \& Goraca, 2015; Ambrosi et al., 2018 and Genazzani et al., 2018). These authors attributed their results to the antioxidant effect of ALA which prevents the hyperlipidemia/hypertensive induced NO and prevents the elevation of serum cytokine. 
Inhibition of an inflammatory mediator TNF- $\alpha$ as well as IL-1 $\beta$ points out an anti-inflammatory effect of ALA against hyperlipidemia/hypertensive induced heart failure. Also, these results are supported by the ability of ALA to prevent the hyperlipidemia/hypertensive necrotic damage in rat hearts and prevention of the increment in the activities of the cardiac enzymes marker CK, $\mathrm{CK}_{-\mathrm{MB}}, \mathrm{LDH}$ and AST.

On the basis of current data, the maximum corrections in all studied biochemical and histological parameters were recorded in hyperlipidemic/hypertensive rats group treated with a mixture of antioxidants CoQ10 and ALA probably due to the synergistic effects of their pharmadynamic and pharmakinetic properties.

From the above cited data, it could be concluded that treatment with CoQ10 or ALA and their mixture can prevent the hyperlipidemia/hypertensive induced oxidative heart damage as well as inflammatory stress in heart by alleviating lipid peroxidation through free radical scavenging or by enhancing the synthesis of antioxidants and improves glutathione redox system which then detoxify free radicals. 


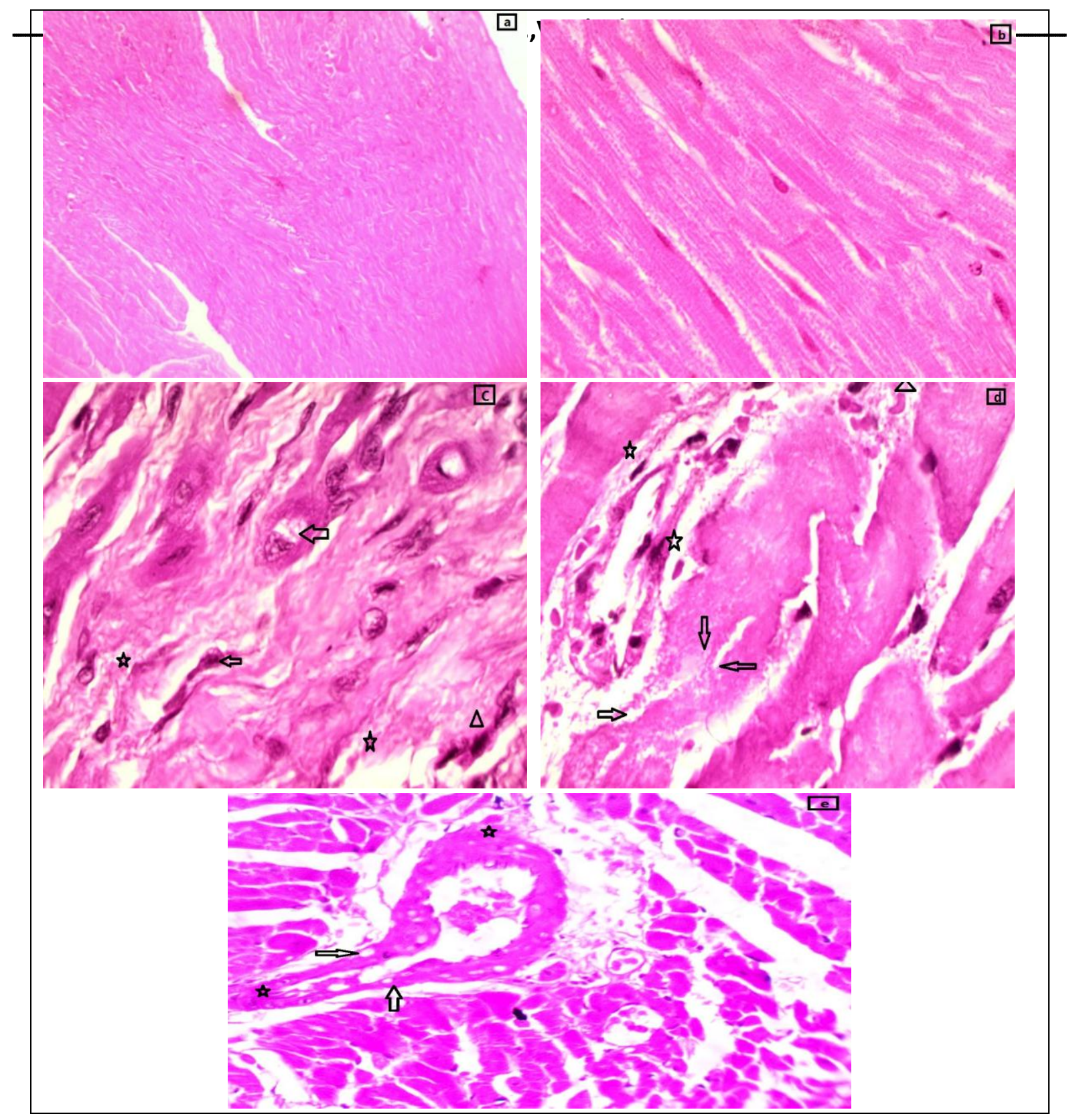

Figure 1: Photomicrographs of sections from control and obese/hypertensive rats (Hx\&E.)

a- Heart section of normal control rat showing normal architecture of cardiac tissue (X 100)

b- Heart section of normal control rats at 4 weeks showing normal architecture of cardiac striations ( $X$ 1000).

c- Heart section of obese/hypertensive rats showing eosinophilic cardiac muscles; lack of striatiour [ ]; groups of musAle fibres [ ] and pyknotic nuclei [ 1000). 
d- Heart section of obese/hypertensive rats showing loss of striation necrosis and lysis [

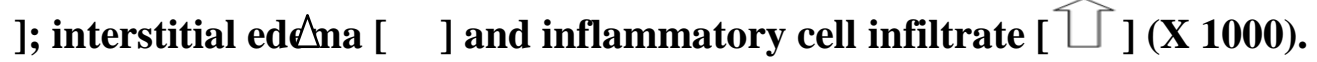

e- Heart section of obese/hypertensive rats showing congested blood vessel infiltrated by fatty degenerative $[\overparen{\square}]$ and reduced tunies media layer $[\mathrm{C}$ (X00)

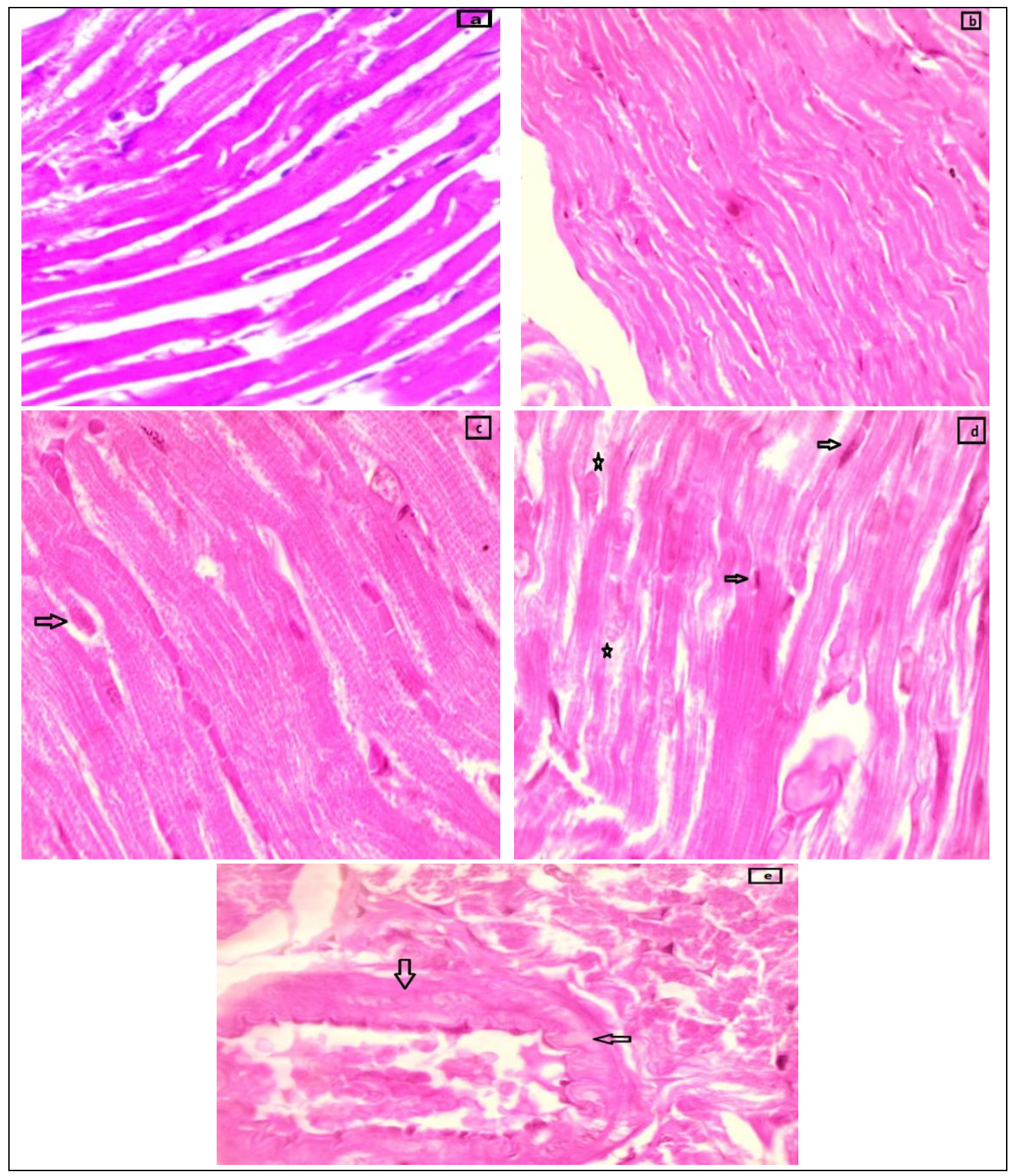

$\Sigma$ 
Figure 2: Photomicrographs of sections from obese/hypertensive rats treated with CoQ10 or/and ALA (Hx\&E.)

a- Heart section of treated rats with CoQ10 showing regenerative profile with surviving muscle fibres (X 400)

b- Heart section of treated rats with ALA showing lesser appearance of degenerative necrotic cardiac cells. (X 400)

c- Heart section of treated rats with CoQ10 and ALA showing cardiac bands with very much attenuated degenerative muscle cells (X 1000)

d- Heart section of treated rats with CoQ10 and ALA showing lesser presence of

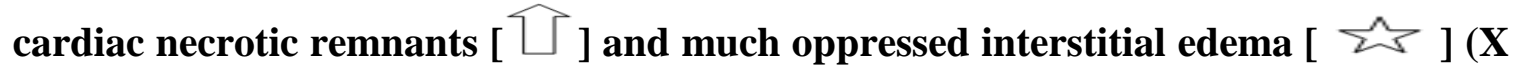
1000)

e- Heart section of treated rats with CoQ10 and ALA showing blood vessels regaining their near to normal appearance $[\square]$ (X 1000) 


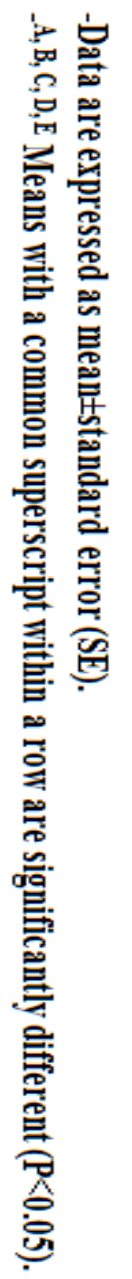

\begin{tabular}{|c|c|c|c|c|c|}
\hline 或 & 西 & $\vec{\Omega}$ & 氙 & & 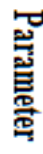 \\
\hline 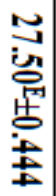 & 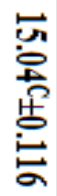 & 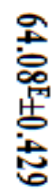 & 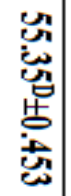 & 2 & \multirow{4}{*}{ 응 } \\
\hline 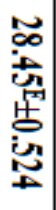 & 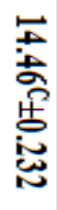 & 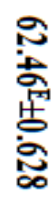 & $\begin{array}{l}\text { 兄 } \\
\text { 苦 } \\
\text { 苦 } \\
\text { 志 }\end{array}$ & $\stackrel{\varrho}{\varrho}$ & \\
\hline 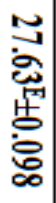 & 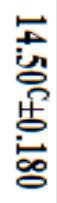 & 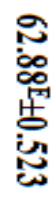 & 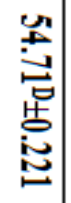 & 蛋 & \\
\hline 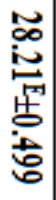 & 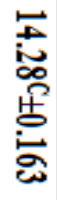 & $\begin{array}{l}\text { 怘 } \\
\text { 兽 } \\
\text { 虫 }\end{array}$ & $\begin{array}{l}\text { 忩 } \\
\text { 品 } \\
\text { 告 } \\
\text { 至 }\end{array}$ & 尝 & \\
\hline 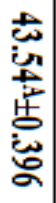 & $\begin{array}{l}\text { 芯 } \\
\text { 总 } \\
\text { 告 } \\
\text { 总 }\end{array}$ & 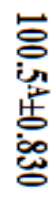 & 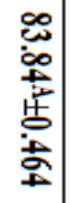 & ஜ & \\
\hline 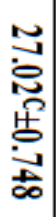 & 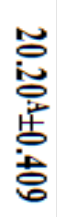 & 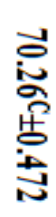 & 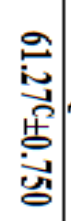 & 응 & 은 \\
\hline 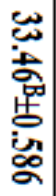 & 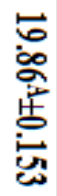 & 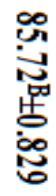 & 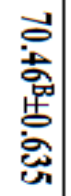 & 点 & \\
\hline 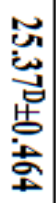 & $\begin{array}{l}\text { 岕 } \\
\text { 兽 } \\
\text { 岕 } \\
\text { 常 }\end{array}$ & $\begin{array}{l}\stackrel{2}{0} \\
\text { 芯 } \\
\text { 告 } \\
\text { 岕 }\end{array}$ & 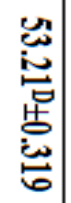 & 尝 & \\
\hline
\end{tabular}

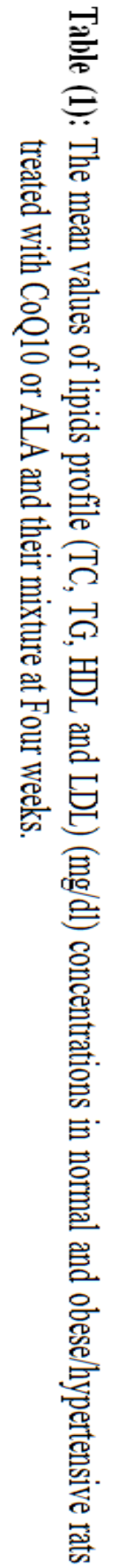



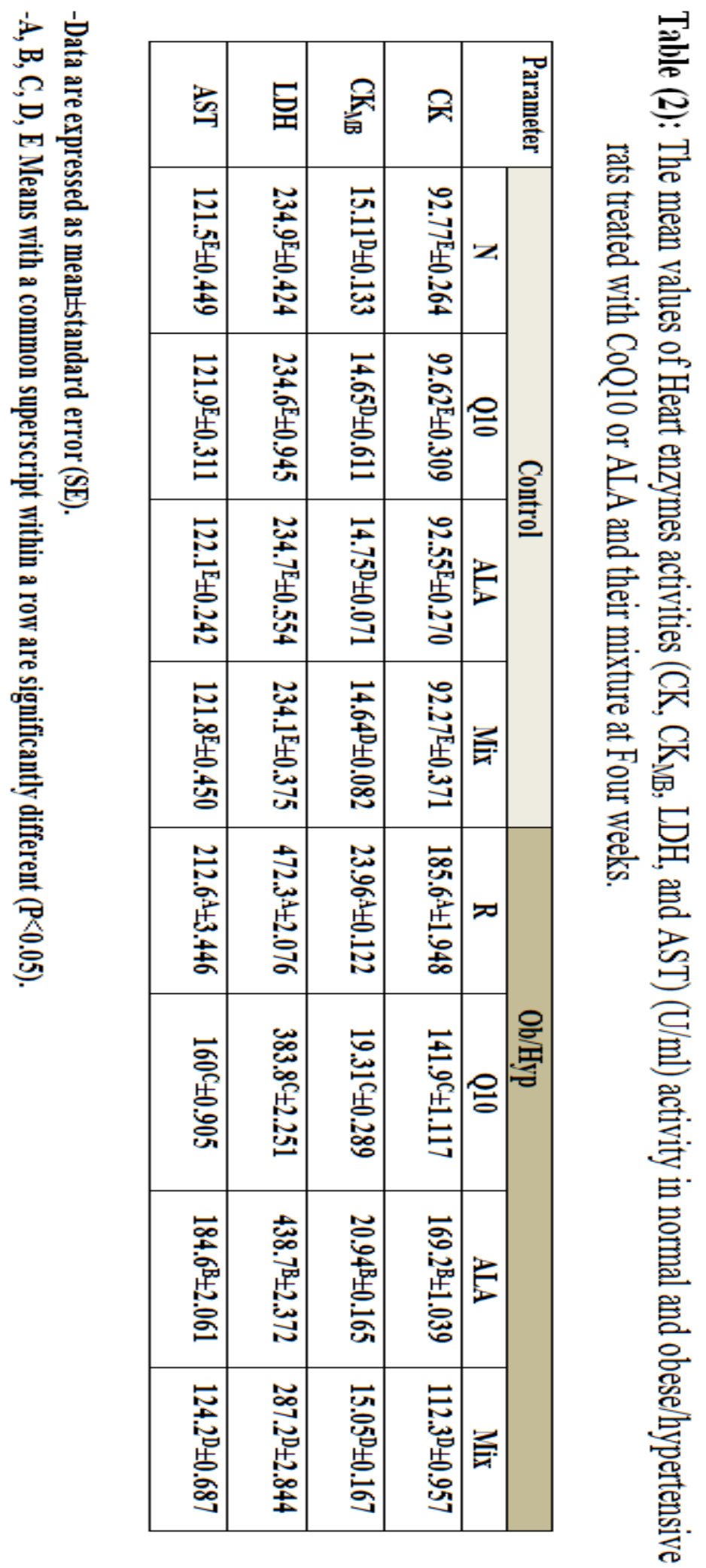


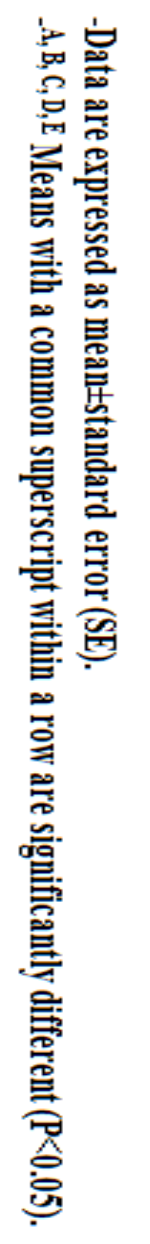

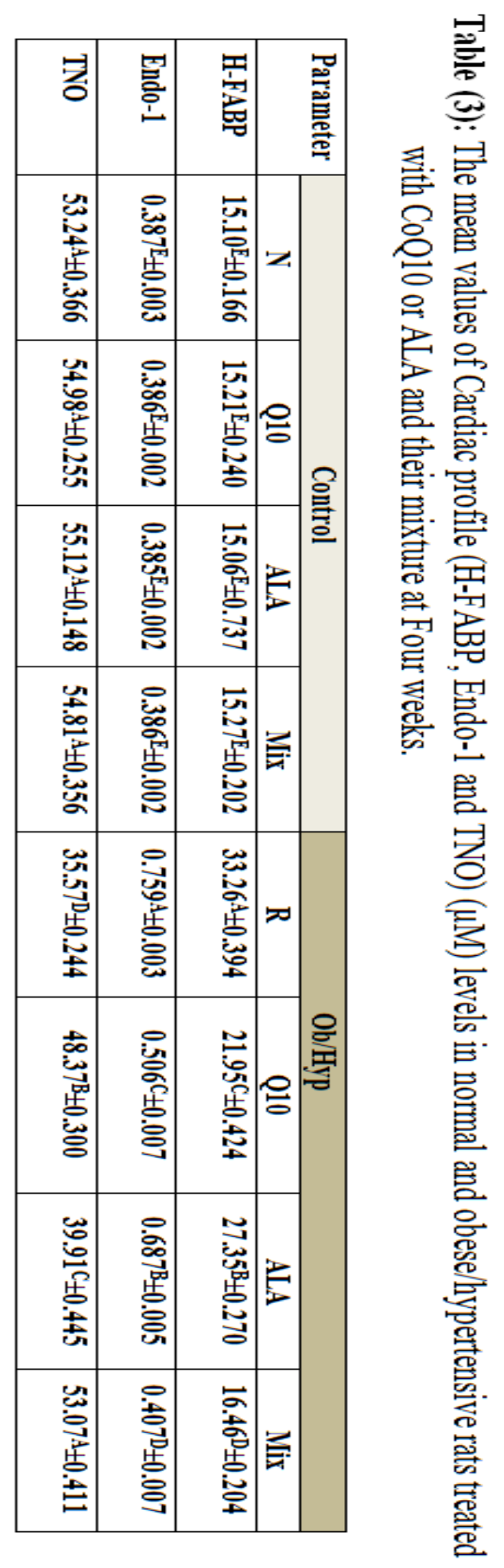




\section{REFERANCES}

Acosta, M.J.; Fonseca, L.V.; Desbats, M.A.; Cerqua, C.; Zordan, R.; Trevisson, E. and Salviati, L.: Coenzyme Q biosynthesis in health and disease. Biochim. Biophys. Acta, 1857(8): 1079-1085(2016).

Allain, C.C.; Poon, L.S.; Chan, C.S.; Richmond, W. and Fu, $\quad$ P.C.: Enzymatic determination of total serum cholesterol. Clin. Chem, 20(4): 470-475(1974).

Ambrosi, N.; Guerrieri, D.; Caro, F.; Sanchez, F.; Haeublein, G.; Casadei, D.; Incardona, C. and Chuluyan, E.: Alpha Lipoic Acid: A Therapeutic Strategy that Tend to Limit the Action of Free Radicals in Transplantation. Int. J. Mol. Sci, 19(1): 102(2018).

Assman, G.; Jabs, H.; Kohnert, U.; Note, W. and Schriewer, H.: The relation between total cholesterol, triglycerides and cholesterol fraction in high and mild lipidemia. Clin. Chem. Acta, 140: 77-83 (1984).

Bekir, M.; Erdal, T. and Sefa, L.: Coenzyme Q10 and Cardiovascular system. Sci. Mov. Health, 15(2): 564-569(2016).

Brahmbhatt, D.H. and Cowie, M.R.: Heart failure:classification and pathophysiology. MEDICINE, 46(10): 587-593(2018).

Brealey, D.; Karyampudi, S.; Jacques, T.S.; Novelli, M.; Stidwill, R.; Taylor, V.; Smolenski, R.T. and Singer, M.: Mitochondrial dysfunction in a long-term rodent model of sepsis and organ failure. Am. J. Physiol. Regul. Integr. Comp. Physiol, 286(3): R491-497(2004).

Buhl, S.N. and Jackson, K.Y.: Optimal conditions and comparison of lactate dehydrogenase catalysis of the lactate-to-pyruvate and pyruvate-tolactate reactions in human serum at 25, 30, and 37 degrees C. Clin. Chem, 24(5): 828-831(1978).

Chaudhary, H.R. and Brocks, D.R.: the single dose poloxamer 407 model of hyperlipidemia; systemic effects on lipids assessed using pharmacokinetic methods, and its effects on adipokines. J. pharm. Pharm. sci, 16 (1): 65-73(2013).

Choi, B.K.; Park, S.B.; Lee, D.R.; Lee, H.J.; Jin, Y.Y.; Yang, S.H. and Suh, J.W.: Green coffee bean extract improves obesity by decreasing body fat in high-fat diet-induced obese mice. ASIAN PAC. J. TROP. MED, 9(7): 635-643(2016).

Faulx, M.D.; Ernsberger, P.; Vatner, D.; Hoffman, R.D.; Lewis, W.; Strachan, R. and Hoit, B.D.: Strain-dependent -adrenergic receptor function influences myocardial responses to isoproterenol stimulation in mice. Am. J. Physiol. Heart Circ. Physiol, 289(1): H30 H36(2005).

Fisher, M.L.; Carliner, N.H. and Becker, L.C.: Serum creatine kinase in the diagnosis of acute myocardial infarction. JAMA, 249(3): 393394(1983). 
Fossati, P.; Burghen, G.A.; Li, H.; Hudson, M.M. and Kun, L.E.: Serum triglycerides determined colourimetrically with an enzyme that produce hydrogen peroxide. Clin. Chem, 28(10): 2077-2080(1982).

Genazzani, A.D.; Shefer, K.; Casa, D.; Prati, A.; Napolitano, A.; Manzo, A.; Despini, G. and Simoncini, T.: Modulatory effects of alpha-lipoic acid (ALA) administration on insulin sensitivity in obese PCOS patients. J. Endocrinol. Invest, 41(5): 583-590(2018).

Goraca, A.; Huk-Kolega, H.; Piechota, A.; Ciejka, E. and Skibska, B.: Lipoic acid biological activity and therapeutic potential. Pharma. Rep, 63(4): 849-858(2011).

Griess, L.C.; Wagner, D.A.; Glogowski, J.; Skipper, P.L.; Wishnok, J.S. and Tannenbaum, S.R.: Analysis of nitrate, nitrite, and $[15 \mathrm{~N}]$ nitrate in biological fluids. Anal. Biochem, 126(1): 131-138(1982).

Griffiths, J. and Handschuh, G.: Creatine kinase isoenzyme MB in myocardial infarction: methods compared. Clin. Chem, 23(3): 567271(1977).

Grove, T.H.: Effect of reagent $\mathrm{pH}$ on determination of HDL cholesterol by precipitation with sodium phosphotungstate-magnesium Clin. Chem, 25(4): 560-567(1979).

Guofu, L.; Linlin, G.; Jia, J.; Xiaoying, G.; Bin, Z. and Weimin, C.:"'alphaLipoic acid prolongs survival and attenuates acute kidney injury in a rat model of sepsis," Clinical and Experimental. Pharmacol. Physiol, 41(7): 459-468(2014).

Heibashy, M.I.A. and Abdel Moneim, A.: Potential benefits of some antioxidant nutrients in reducing the high levels of some biochemical variables associated with induced hypertension in rats. Isotope. Rad. Res, 37(2): 465-479(2005).

Heibashy, M.I.A. and Mazen, G.M.A.: Hyperhomocysteinemia is a key for aggravation of liver injury in non-alcoholic fatty liver disease associated with cardiovascular disease in rats. Int. J. Curr. Adv. Res, 2(9): 161-176(2014).

Heibashy, M.I.A.; Mazen, G.M.A. and Shahin, M.I.: The Curative Effects of some Antioxidants on Endotoxin Induced with Lipopolysaccharide in the Liver of Rats. J. Am. Sci, 9(12): 529-538(2013).

Heibashy, M.I.A.; Zaki, N.A. and El-Nahrawy, W.A.M.: Alterations in Insulin, Leptin, Orexin-A andNeuropeptide-Y Levels in the High-Fat Diet Fed Rats. IJBAS, 4(6): 1473-1481(2010).

Hodgson, J.M. and Watts, G.F.: Can coenzyme Q\{10\} improve vascular function and blood pressure? Potential for effective therapeutic reduction in vascular oxidative stress. BIOFACTORS, 18(1-4): 129-136(2003). 
Hulanicka, B.; Lipowicz, A.; Koziel, S. and Kowalisko, A.: Relationship between early puberty and the risk of hypertension/overweight at age 50: evidence for a modified Barker hypothesis among Polish youth. ECON. HUM. BIOL, 5(1): 48-60(2007).

IFCC: Expert Panel on enzyme of The IFCC, Clin. Chim. Acta, 70: F19F21(1978).

Karri, S.; Sharma, S.; Hatware, K. and Patil, K.: Natural anti-obesity agents and their therapeutic role in management of obesity: A future trend perspective. BIOMED. PHARMACOTHER, 110: 224-238(2019).

Khallaf, H.A. and El-Mansy, A.A.R.: The possible alleviating effect of saffron on chlorpyrifos experimentally induced cardiotoxicity: Histological, immunohistochemical and biochemical study. Acta. Histochemica, 121(4): 472-483(2019).

Khatta, M.; Alexander, B.S.; Krichten, C.M.; $\quad$ Fisher, M.L.; Freudenberger, R.; Robinson, S.W. and Gottlieb, S.S.: The effect of coenzyme Q10 in patients with congestive heart failure. Ann. Intern. Med, 132(8): 636-640(2000).

Kitada, K.; Daub, S.; Zhang, Y.; Klein, J.D.; Nakano, D.; Pedchenko, T.; Lantier, L.; LaRocque, L.M.; Marton, A.; Patrick Neubert, P.; Schröder, A.; Rakova, N.; Jantsch, J.; Dikalova, A.E.; Dikalov, S.I.; Harrison, D.G.; Müller, D.N.; Akira Nishiyama, A.; Rauh, M.; Harris, R.C.; Luft, F.C.; Wasserman, D.H.; Sands, J.M. and Jens Titze, J.: 'High salt intake reprioritizes osmolyte and energy metabolism for body fluid conservation'. JCI, 127(5): 1944-1959(2017).

Klotz, S.; Hay, I.; Zhang, G.; Maurer, M.; Wang, J. and Burkhoff, D.: Development of Heart Failure in Chronic Hypertensive Dahl Rats Focus on Heart Failure with Preserved Ejection Fraction. Hypertens, 47(5): 901-911(2006).

Korolenko, T.A.; Johnston , T.P.; Dubrovina, N.I.; Kisarova, Y.A.; Zhanaeva, S.Y.; Cherkanova, M.S.; Filjushina, E.E.; Alexeenko, T.V.; Eva Machova, E. and Zhukova, N.A.: Effect of poloxamer 407 administration on the serum lipids profile, anxiety level and protease activity in the heart and liver of mice. Interdiscip. Toxicol, 6(1): 1825(2013).

Korolenko, T.A.; Johnston, T.P.; Tuzikov, F.V.; Tuzikova, N.A.;

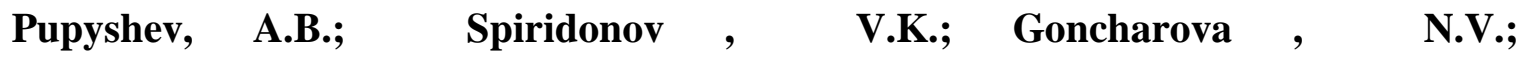
Maiborodin, I.V. and Zhukova, N.A.: Early-stage atherosclerosis in poloxamer 407-induced hyperlipidemic mice: pathological features and changes in the lipid composition of serum lipoprotein fractions and subfractions. Lipid Health Dis, 15(1): 96-101(2016). 
Korolenko, T.A.; Tuzikov, F.V.; Johnston, T.P.; Tuzikova, N.A.; Ya, k. and Ya, Z.: The influence of repeated administration of poloxamer 407 on serum lipoproteins and protease activity in mouse liver and heart. Can. J. Physiol. Pharmacol, 90(11): 1456-1468(2012).

Krishnan, S.; Sinha, P. and Patil, S.: Successful delivery in a morbidly obese patient after failed intubation and regional technique. Br. J. Anaesth. 99(6): 919-920(2007).

Lanaspa, M.A.; Kuwabara, M.; Andres-Hernando, A.; Li, N.; Cicerchi, C.; Jensen, T.; Orlicky, D.J.; Roncal-Jimenez, C.A.; Ishimoto, T.; Nakagawa, T.; Rodriguez-Iturbe, B.; MacLean, P.S. and Johnson, R.J.: High salt intake causes leptin resistance and obesity in mice by stimulating endogenous fructose production and metabolism. PNAS, 115(12): 3138-3143(2018).

Lau, J.L. and Dunn, M.K.: Therapeutic peptides: Historical perspectives, current development trends, and future directions. BIOORGAN. MED. CHEM, 26(10): 2700-2707(2018).

Littarru, G. P.: Effect of coenzyme Q10 administration on endothelial function and extracellular superoxide dismutase in patients with ischaemic heart disease: a double-blind, randomized controlled study. Eur. Heart J, 28(18): 2249-2255(2007).

Madamanchi, N.R. and Runge, M.S.: "Redox signaling in cardiovascular health and disease". Free. Rad. Biol. Med, 61: 473-501(2013).

Mahmoud, A.M.; Szczurek, M.R.; Blackburn, B.K.; Mey, J.T.; Chen, Z.; Robinson, A.T.; Bian, J.T.; Unterman, T.G.; Minshall, R.D.; Brown, M.D.; Kirwan, J.P.; Phillips, S.A. and Haus, J.M.: Hyperinsulinemia augments endothelin-1 protein expression and impairs vasodilation of human skeletal muscle arterioles. Physiol. Rep, 4(16): e12895(2016).

McMurray, J.J.; Dunselman, P.; Wedel, H.; Cleland, J.G.; Lindberg, M.; Hjalmarson, A.; Kjekshus, J.; Waagstein, F.; Apetrei, E.; Barrios, V.; Böhm, M.; Kamenský, G.; Komajda, M.; Mareev, V. and Wikstrand, J.: CORONA Study Group. Coenzyme Q10, rosuvastatin, and clinical outcomes in heart failure: a pre-specified substudy of CORONA (Controlled Rosuvastatin Multinational Study in heart failure). J. Am. Coll. Cardiol, 56: 1196-1204(2010).

Özkan, Y.; Yilmaz, O.; Öztürk, A.I. and Erşan, Y.: Effects of triple antioxidant combination (vitamin $\mathrm{E}$, vitamin $\mathrm{C}$ and $\alpha$-lipoic acid) with insulin on lipid and cholesterol levels and fatty acid composition of brain tissue in experimental diabetic and non-diabetic rats. Cell Biol. Int, 29(9): 754-760(2005). 
Packer, L. and Cadenas, E.: Text book of Method in enzymology Nitric oxide, part $\mathrm{F}$ Oxidative and Nitrosative stress in redox regulation of cell signaling, Los Angeles, 48: 26-32(2011).

Pagani, F.; Bonora, R.; Bonetti, G. and Panteghini, M.: Evaluation of a sandwich enzyme-linked immunosorbent assay for the measurement of serum heart fatty acid-binding protein. Ann. Clin. Biochem, 37(4): 404405(2002).

Papanas, N. and Ziegler, D.: Efficacy of $\alpha$-lipoic acid in diabetic neuropathy. Expert. Opin. Pharmacother, 15(18): 2721-2731(2014).

Ramanathan, V. and Thekkumalai, M.: Role of chrysin on hepatic and renal activities of N $\omega$ nitro-l-arginine-methylester induced hypertensive rats. Int. J. nutr. Pharma. neuro. disease, 4(1): 58-63(2014).

Rana, M.G.; Dudhrejiya, A.V. and Katbamna, R.V.: antihyperlipidemic and antioxidant activity of biofractions of flowers of hibiscus rosa-sinensis in poloxamer 407 induced hyperlipidemic rats. W. J. Pharma. Pharma. Sci., 6(7): 1665-1682(2017).

Rochettea, L.; Lorina, J.; Zellera, M.; Guillanda, J.C.; Lorgisab, L.; Cottin, y. and Vergelya, C.: Nitric oxide synthase inhibition and oxidative stress in cardiovascular diseases: Possible therapeutic targets?. PHARMACOL. THERAPEUT, 140(3): 239-257(2013).

Rodick, T.C.; Seibels, D.R.; Babu, J.R.; Huggins, K.W.; Ren, G and Mathews, S.T.: Potential role of coenzyme Q10 in health and disease conditions. Nutr. Diet. Supp, 10: 1-11(2018).

Saidua, Y.; Usmana, M.J.; Isaa, S.A.; Isezuob, S.A.; $\quad$ Bilbisa, L.S.; Sahabic, S.M.; Bellod, A. and Muhammada, S.A.: Biochemical and histological changes in the heart of post-partum rats exposed to Natron. IHJ, 70(6): 887-893(2018).

Samavat, T.; Hojjatzade, A.; Shams, M.; Afkhami, A.; Mahdavi, A.; Bashti, S.H.; Pouraram, H.; Ghotbi, M. and Rezvani, A.: Prevention and control of cardiovascular disease (for government employees). Second edition(2012).

Sander, S.; Coleman, C. I.; Patel, A. A.; Kluger, J.; and White, C.M.: The impact of coenzyme Q10 on systolic function in patients with chronic heart failure. J. Card. Fail, 12(6): 464-472(2006).

Sharma, A.; Fonarow, G.C.; Butler, J.; Ezekowitz, J.A. and Felker, G.M.: Coenzyme Q10 and Heart Failure: A State-of-the-Art Review. Circ. Heart Fail, 9(4): e002639(2016). 
Simonds, S.E.; Pryor, J.T.; Ravussin, E.; Greenway, F.L.; Dileone, R.; Allen, A.M.; Bassi, J.; Elmquist, J.K.; Keogh, J.M.; Henning, E.; Myers, M.G. J.; Licinio, J.; Brown, R.D.; Enriori, P.J.; O'Rahilly, S.; Sternson, S.M.; Grove, K.L.; Spanswick, D.C.; Farooqi, I.S. And Cowley, M.A.: Leptin mediates the increase in blood pressure associated with obesity. Cell, 159(6):1404-1416(2014).

Skibska, B. and Goraca, A.: The Protective Effect of Lipoic Acid on Selected Cardiovascular Diseases Caused by Age-Related Oxidative Stress Oxidative. Oxid. Med. Cell Longev, 2015: 313021(2015).

Song, X.; Qu, H.; Yang, Z.; Rong, J.; Cai, W. and Zhou, H.: Efficacy and Safety of L-Carnitine Treatment for Chronic Heart Failure: A MetaAnalysis of Randomized Controlled Trials. Biomed. Res. Int, 2017: 6274854(2017).

SPSS.: IBM SPSS Statistics version 20. Chicago (IL) (2009).

Tanaka, H.; Ishida, T.; Johnston, T.P.; Yasuda, T.; Ueyama, T. and Kojima, Y.: Role of endothelial lipase in plasma HDL levels in a murine model of hypertriglyceridemia. $\mathrm{J}$. Atheroscler. Thromb., 16(4): 327-338(2009).

Tiano, L. and Littarru, G.P.: Bioenergetic and antioxidant properties of coenzyme Q10: recent developments. Mol. Biotechnol, 37(1): 317(2007).

Tsai, K.L.; Huang, Y.H.; Kao, C.L.; Yang, D.M.; Leed, H.C.; Choue, H.C.; Chen, Y.C.; Chiou, G.Y.; Chen, L.H.; Yang, Y.P.; Chiu, T.H.; Tsai, C.S.; Ou, H.C. and Chiou, S.H.: A novel mechanism of coenzyme Q10 protects against human endothelial cells from oxidative stress-induced injury by modulating NO-related pathways. J. Nutr. Biochem, 23 (5): 458-468(2012).

Tsuneki, H.; Sekizaki, N.; Suzuki, T.; Kobayashi, S.; Wada, T. and Okamoto, T.: Coenzyme Q10 prevents high glucose-induced oxidative stress in human umbilical vein endothelial cells. Eur. J. Pharmacol, 566(1-3):1-10(2007).

Vanhoutte, P.M.: Nitric Oxide: From Good to Bad. Ann. Vasc. Dis, 11(1): 4151(2018).

Venkat, R.D.; Chandraiah, G.; Meena, $\quad$ A. $\quad$ K.; $\quad$ Kumar, $\quad$ M. $\quad$ N. $\quad$ V and Ramarao, P.: The Co-Encapsulated Antioxidant Nanoparticles of Ellagic Acid and Coenzyme Q10 Ameliorates Hyperlipidemia in High Fat Diet Fed Rats. J. Nano. Nanote, 9(11): 6741-6746(2009).

Vupputuri, A.; Sekhar, S.; Krishnan, S.; Venugopal, K. and Natarajane, K.U.: Heart-type fatty acid-binding protein $(\mathrm{H}-\mathrm{FABP})$ as an early diagnostic biomarker in patients with acute chest pain. Indian Heart J, 67(6): 538-542(2015). 
Wakisaka, S; Terui, N. and Suzuki H.: Endothelin-1 kinetics in plasma urine, and blister fluid in burn patients. Ann. Plast. Surg, 37(3): 305309(1996).

WHO.: Obesity and overweight. Geneva, World Health Organization (to be published on 16 February 2018)(2017).

Willemsen, R.T.; van Severen, E.; Vandervoort, P.M.; Grieten, L.; Buntinx, F.; Glatz, J.F. and Dinant, G.J.: Heart-type fatty acid binding protein (H-FABP) in patients in an emergency department setting, suspected of acute coronary syndrome: optimal cut-off point, diagnostic value and future opportunities in primary care. Eur. J. Gen. Pract, 21(3): 156-63(2015).

Yang, Y.K.; Wang, L.P.; Chen, L.; Yao, X.P.; Yang, K.Q.; Gaod, L.G. and Zhou, X.L.: Coenzyme Q10 treatment of cardiovascular disorders of ageing including heart failure, hypertension and endothelial dysfunction. Clinica. Chimica. Acta, 450(23): 83-89(2015).

Yi, X. and Maeda, N.: Endogenous Production of Lipoic Acid Is Essential for Mouse Development. Mol. Cell Biochem, 25(18): 8387-8392(2005).

Youshionwa, K.; Terada, N.; Matsui, Y.; Terai, A.; Kinukawa, N. and Arai, Y.: Prevalence of and risk factors for nocturia: analysis of a health screening program. Int. J. Urol, 11: 282-287(2004).

Zhang, J.C.; Yu, X.Y.; Huang, Y.; Yung, L.M.; Lau, C.W. and Lin, S.G.: Apelin modulates aortic vascular tone via endothelial nitric oxide synthase phosphorylation pathway in diabetic mice. Cardio. Res, 74 (3): 388-395(2007).

Ziegler, D.; Low, P.A.; Litchy, W.J.; Boulton, A.J.; Vinik, A.I.; Freeman, R.; Samigullin, R.; Tritschler, H.; Munzel, U.; Maus, J.; Schütte, K. and Dyck, P.J.: 60Efficacy and safety of antioxidant treatment with $\alpha$-lipoic acid over 4 years in diabetic polyneuropathy: the NATHAN 1 trial. Diabetes Care, 34(9): 2054-60(2011). 


\section{الملخص باللغة العربية}

\section{الدور العلاجى لأنزيم كيو- · 1 أو/و حمض ألفا الليبوليك على وظائف القلب فى الجرذان البدينة ومرتفعة ضغط الام.}

منى فتحى شوقى نصر ا ، سناء محمد رفعت ا، محمد اسلام حبيشى ب

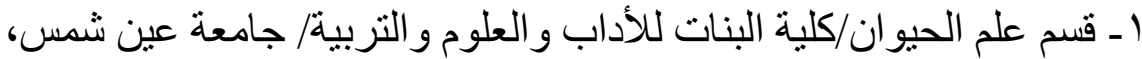
Y ـ مركز البحوث النووية/ هيئة الطاقة الذرية.

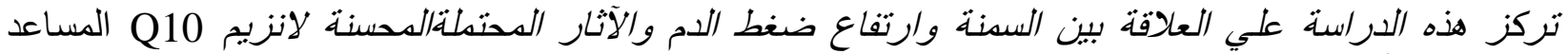

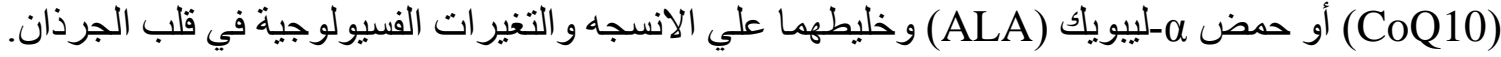

ولتحقيق هذا الغرض، أجريت مقارنه بين مجموعه فئران المجموعة الضابطة (·r جرذا) ومجموعه فئران

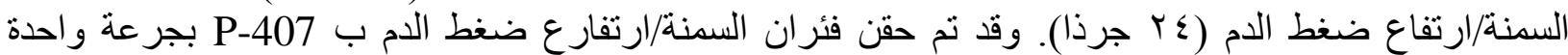

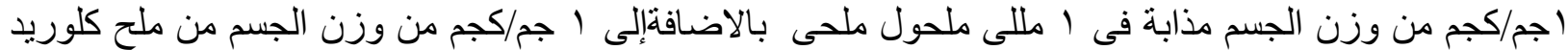

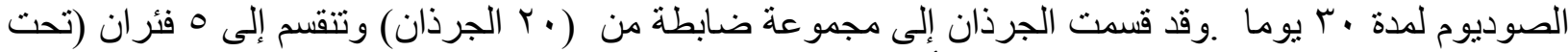

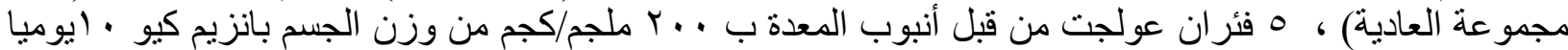

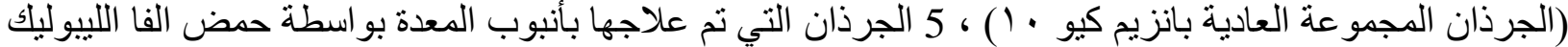

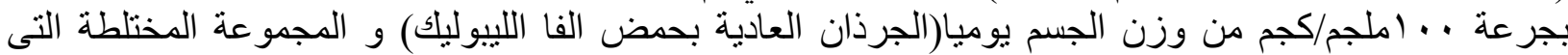

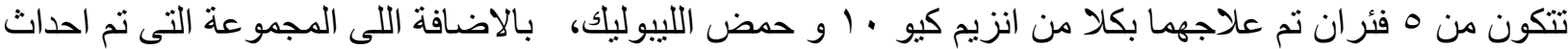

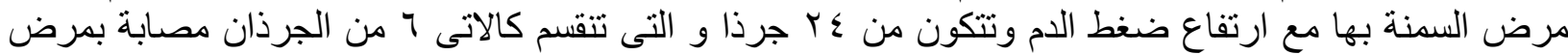

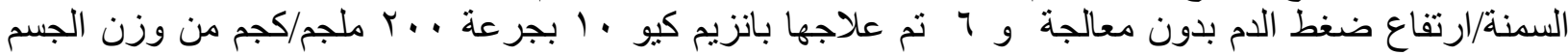

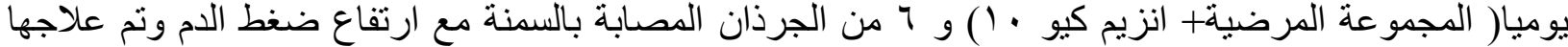

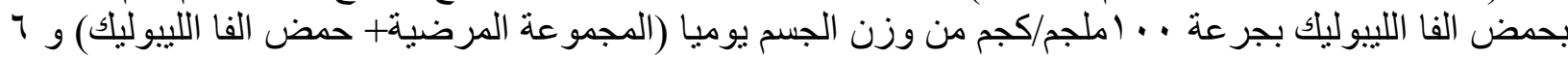

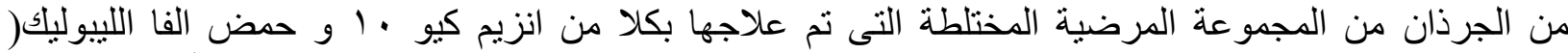

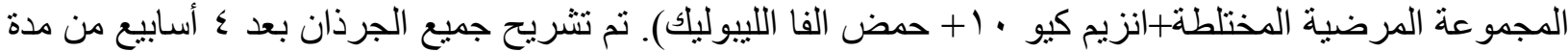
التجربة.

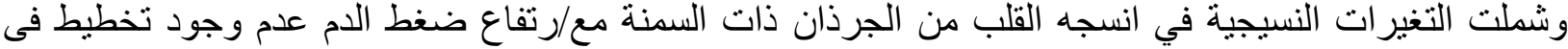

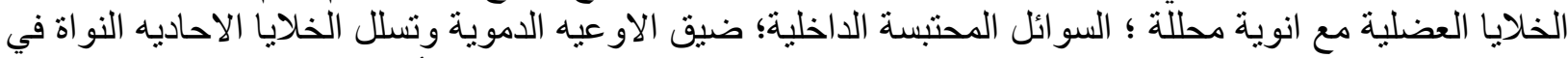

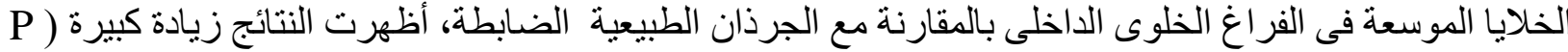

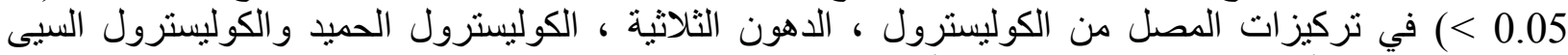

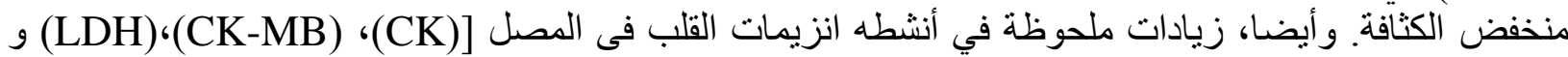
[(AST)

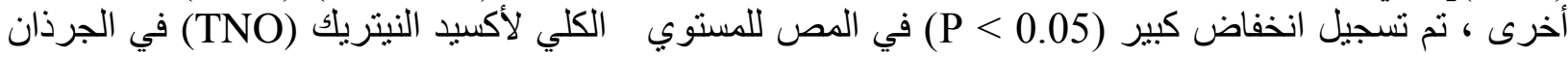

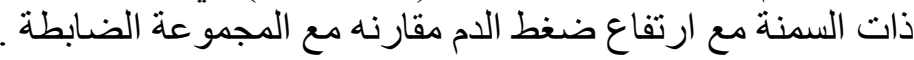

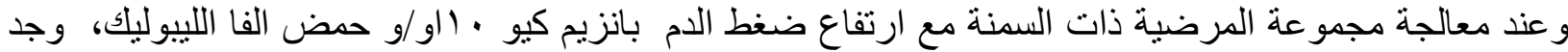

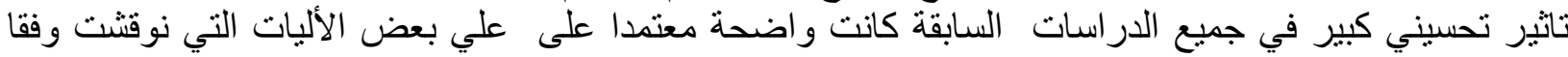

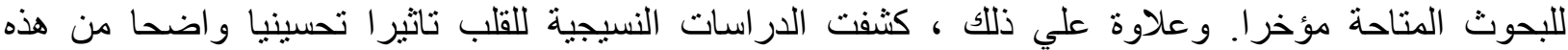

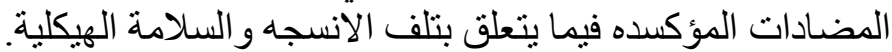

